\title{
Integrability of the KdV hierarchies via group theoretical approach
}

\author{
Zainal Abdul Aziz* \\ Ibnu Sina Institute for Fundamental Science Studies, Universiti Teknologi Malaysia, 81310 UTM Skudai, Johor, Malaysia \\ *To whom correspondence should be addressed. E-mail: zaa@mel.fs.utm.my
}

Received 10 January 2007

http://dx.doi.org/10.11113/mjfas.v3n1.22

\begin{abstract}
This paper explores the integrability of the Korteweg-de Vries (KdV) hierarchies via a renowned group theoretical approach. Briefly this work employs the group representation method in identifying a (physically significant) nonlinear dynamical system as an integrable Hamiltonian system.

| Integrability | KdV hierarchy | Group theoretical approach |
\end{abstract}

\section{Introduction}

Extensive studies on group representations and the foundations of mechanics of recent years have found that the dual space $\boldsymbol{g}^{*}$ to the Lie algebra $\boldsymbol{g}$ of a Lie group $\boldsymbol{G}$, supports a natural Poisson structure (refer Weinstein [23], Schmid [18]). We consider here that the form of the Poisson structure of the group theoretical approach (primarily related to works by Kirillov [12], Konstant [13], Souriau [21]) represents the symplectic structure of the KdV hierarchy's Hamiltonian system. This is obtainable from $\boldsymbol{g}^{*}$ of an infinite dimensional Lie algebra $\boldsymbol{g}$ algebra of formal pseudo differential operator of negative degree. Subsequently the KdV hierarchy acts as a Hamiltonian system on a coadjoint orbit of the Lie algebra dual space of the pseudo differential operator. Recent research works (refer Drinfeld \& Sokolov [8], Sato [18], Frenkel [9]) based on similar arguments have successfully shown that intrinsically certain integrable systems or categorically Zakharov-Shabat-AblowitzKaup-Newell-Segur (ZS-AKNS) system acts as a Hamiltonian system on a coadjoint orbit of the affine Lie algebra dual space.

Interestingly, within the framework of Lax approach and Gelfand-Dickey programme, the Lax pairs represent the Hamiltonian system and thus the pairs can reside and operate in $\boldsymbol{g}$. Strictly speaking, this formulation exhibits a coherent connection between Lax representation and mechanics on the coadjoint orbit space of a Lie group action. This manifestation would naturally explain our motive to exhibit the integrability of the KdV hierarchies via this group theoretical approach.

This paper is organized as follows: section 2 briefly describes the Gelfand-Dickey programme, section 3 outlines concisely the meaning of integrability in this formulation by considering two relevant theorems, and section 4 
tersely explores the integrability of the KdV hierarchies (or simply the system of generalised KdV equation) by reworking on two important propositions. Finally we end this paper with some comments on this approach.

\section{The Gelfand-Dickey Programme}

Gelfand and Dickey [11] developed the algebra and variational calculus in the ring of polynomial functions of $\varphi_{k} \in \mathrm{H}_{S, T}$ and their derivatives with respect to $x$, where $\mathrm{H}_{S, T}$ is the function space with integers $S \geq 0, T \in R$. Lax [14] earlier expressed the $\mathrm{KdV}$ in the form $L_{t}=[L, B] ; \quad L=-\partial_{x}^{2}+\varphi(x, t)$ and $B=4\left(\partial_{x}^{3}+\chi \partial_{x}+\partial_{x} \cdot \chi\right)$ with $\chi=\frac{-3}{4} \varphi$. Obviously we have the equivalence between $\varphi_{t}=X_{H_{j}}(\varphi)$ and $L_{t}=\left[B_{m}, L\right] ; j \in Z_{+}, m$ any integer or zero. $B_{m}$ an antisymmetric differential operator. Generally, if

$$
L=\sum_{k=0}^{n} \varphi_{k}(x, t)\left(-i \partial_{x}\right)^{k}
$$

$\varphi_{n} \equiv 1, \varphi_{n-1} \equiv 0, \varphi_{k} \in H_{S, T}, \quad i=\sqrt{-1}$, then we can obtain a suitable differential operator $B_{m}$ of order $2 m+1$ such that the commutator bracket $\left[B_{m}, L\right]$ is a differential operator of order $(n-2)$, i.e.

$$
B_{m}=\partial_{x}^{2 m+1}+\sum_{j=1}^{m}\left\{\chi_{j} \partial_{x}^{2 j-1}+\partial_{x}^{2 j-1} \cdot \chi_{j}\right\}
$$

By using the symbolic technique and expressions involving the resolvent diagonal and fractional powers to $L$, Gelfand and Dickey [10] were able to show the Lax representation in the 'symbol space'; i.e.

$$
\begin{aligned}
L_{t}(\xi) & =\left[B_{m}(\xi, x), L(\xi)\right] \\
& =\sum_{\ell=N+1}^{\infty} \sum_{v=0}^{\infty} \sum_{k=0}^{n}(-i)^{v} K\left(\varphi_{k}, \varphi_{k, x}, \ldots, \varphi_{k, x^{v}}, \ldots\right) \xi^{N-\ell+k-v},
\end{aligned}
$$

which is equivalent to the system of equations

$$
\varphi_{r, t}=\sum_{\substack{N-\ell+k-v=r \\(\ell>N)}}(-i)^{v} K\left(\varphi_{k}, \varphi_{k, x}, \ldots, \varphi_{k, x^{v}}, \ldots\right) ; r=0,1, \ldots, n-2
$$

where $K$ a polynomial function. Essentially equation (1) embodies the KdV hierarchies or the system of generalised $\mathrm{KdV}$ equation. In addition, it can be shown (refer Gelfand and Dickey [10]) that the KdV hierarchies are equivalent to the Hamiltonian equations

$$
\widetilde{\varphi}_{t}=X_{H_{j}}(\varphi) ; \quad \widetilde{\varphi}=\left(\varphi_{0}, \varphi_{1}, \ldots, \varphi_{n-2}\right)
$$

since (1) can be written as 


$$
\varphi_{t}=J \frac{\delta H}{\delta \widetilde{\varphi}}, \quad \frac{\delta}{\delta \widetilde{\varphi}}=\left(\frac{\delta}{\delta \varphi_{0}}, \ldots, \frac{\delta}{\delta \varphi_{n-2}}\right)
$$

$G_{H_{j}}(\widetilde{\varphi})=\frac{\delta H_{j}}{\delta \widetilde{\varphi}}=\sum_{k=0}^{\infty}\left(-\partial_{x}\right)^{k} \frac{\delta H_{j}}{\delta \widetilde{\varphi}_{x^{k}}}, \quad$ the variational derivative of $H$ (representing the gradient of $H_{j}$ ) and $J$ the skew symmetric matrix consisting of the differential operator of the form

$$
J_{r s}=\sum_{\gamma=0}^{n-1-r-s}\left[\left(\begin{array}{c}
\gamma+r \\
r
\end{array}\right) \varphi_{r+s+\gamma+1}\left(-i \partial_{x}\right)^{\gamma}-\left(\begin{array}{c}
\gamma+s \\
s
\end{array}\right)\left(i \partial_{x}\right)^{\gamma} \varphi_{r+s+\gamma+1}\right]
$$

If $H_{j}, H_{k} \in C / D C$ (C, a ring of polynomial function $\varphi_{k}(x), \varphi_{k, x}(x), \ldots$ modulo $D C, D$ differential ring), $j, k \in Z_{+}$are two functionals over $C$, then $J$ would define the Poisson bracket

$$
\left\{H_{j}, H_{k}\right\}=\int_{R} \sum_{r, s=0}^{n-2} \frac{\delta H_{j}}{\delta \varphi_{r}}\left[J_{s r} \frac{\delta H_{k}}{\delta \varphi_{s}}\right] d x
$$

and interestingly Gelfand and Dickey [10] had shown that all the first integrals $H_{j}$ of the system (2) would form the involutive system, i.e.

$$
\left\{H_{j}, H_{k}\right\}=0, \quad \forall j, k \in Z_{+}
$$

\section{Concept of integrability in this formulation}

Dynamical formulation in this framework is based on the following facts:

A. Infinite dimensional representation theory for each Lie $\boldsymbol{G}$ is well connected to a finite dimensional representation of $\boldsymbol{G}$. The representation acts in the dual space $\boldsymbol{g} *$ to $\boldsymbol{g}$ for $\boldsymbol{G}$ and is called the coadjoint representation.

B. Orbit of a Lie group in a space of coadjoint representation represents a symplectic manifold and can be interpreted as phase space of a system of Hamiltonian mechanics. The Lie group is a symmetry group.

The splitting of a Lie algebra into a direct sum of vector spaces for several Lie algebras is found (Konstant [13], Symes [22]) to be responsible for the integrability of a Hamiltonian system with Lax representation. The Konstant-Symes theorem discusses elaborately this key aspect.

Konstant-Symes Theorem (refer Adler \& van Moerbeke [2])

Let $\boldsymbol{g}$ be a Lie algebra having a direct sum decomposition of vector spaces as follows

$$
\boldsymbol{g}=\boldsymbol{h} \oplus \boldsymbol{k},
$$


$\boldsymbol{h}$ and $\boldsymbol{k}$ are Lie subalgebras. Assume that $\boldsymbol{g}$ can be identified with its dual vector space, $\boldsymbol{g} \boldsymbol{*}$ via a nondegenerate bilinear form and $a d$-invariant $(\cdot, \cdot)$. Subsequently it is induced on $\boldsymbol{g} *$ a direct sum decomposition

$$
\boldsymbol{g}^{*}=\boldsymbol{h}^{\perp} \oplus \boldsymbol{k}^{\perp},
$$

The nondegeneracy of $(\cdot, \cdot)$, would naturally derive the association of $\boldsymbol{h}^{\perp} \sim \boldsymbol{k}^{*}$ and $\boldsymbol{k}^{\perp} \sim \boldsymbol{h}^{*}$; whereby if $\boldsymbol{h}^{\perp}$ were to be identified with $\boldsymbol{k}^{*}$, then it inherits a symplectic structure on the coadjoint orbit of $A \in \boldsymbol{k}^{\propto}, \Theta_{A}$. Let $\boldsymbol{k}^{\propto} \subset \boldsymbol{h}^{\perp}$ is an invariant submanifold with respect to the coadjoint action $\boldsymbol{k}$ on $\boldsymbol{h}^{\perp} \sim \boldsymbol{k}^{*}$, and suppose $\boldsymbol{a}\left(\boldsymbol{k}^{\propto}\right)$ is an algebra of functions in $S(\boldsymbol{g} *)$ (or at least defined on a neighbourhood of $\boldsymbol{k}^{\propto}$ ) which is $A d *$ invariant on $\boldsymbol{g} *$. When the functions are restricted to $\boldsymbol{k}^{\propto}, \boldsymbol{a}\left(\boldsymbol{k}^{\propto}\right)$ forms an involutive system; i.e. a system with commutative integrals of Poisson type on $\boldsymbol{k}^{\propto}$ or on the orbit $\boldsymbol{k}^{\propto}$, via the symplectic structure orbit. In addition, if $H_{j} \in \boldsymbol{a}\left(\boldsymbol{k}^{\propto}\right)$ then the Hamiltonian vector fields $X_{H_{j}}(A) \in T_{A} \boldsymbol{k}^{\propto} ; A \in \boldsymbol{k}^{\propto}$, can be written as $X_{H_{j}}(A)=a d_{B_{j}}^{*} A \quad ; j \in Z_{+}$

$A \in \boldsymbol{k}^{\propto} \subset \boldsymbol{h}^{\perp}, B_{j}=-\prod_{\boldsymbol{h}} \boldsymbol{G}_{\boldsymbol{H}_{j}}(A) \in \boldsymbol{h} ; \prod_{h}$ a canonical projection which maps $\boldsymbol{g}$ and $\boldsymbol{g} *$ into $\boldsymbol{h}$ along $\boldsymbol{k}$; $G_{H} \in S\left(g^{*}\right), G_{H}=G_{H_{h^{\perp}}} \oplus G_{H_{\mid k^{\perp}}} ; G_{H_{h^{\perp}}}=\prod_{k} G_{h} \in k, G_{H_{\mid k^{\perp}}}=\prod_{h} G_{h} \in h$

This means that the system of Hamiltonian equations take the form of $A_{t}=a d_{B}^{*} A$

If $(\cdot, \cdot)$ is symmetry for $\boldsymbol{g} \sim \boldsymbol{g}^{*}$ and $a d *=-a d$, then $(*)$ and $(* *)$ can be represented by the Lax equation $A_{t}=X_{H_{j}}(A)=\left\lfloor A, B_{j}\right\rfloor$.

\section{Remark 1}

We discuss here several important remarks that can be concluded from the above theorem:

a. The coadjoint orbit $\boldsymbol{k}^{\propto}$ of the dual space algebra Lie $\boldsymbol{g}$ represents the 'phase space' of a system of Hamiltonian mechanics and certainly the Lax pairs naturally live in $\boldsymbol{g}$.

b. The coadjoint orbit $\boldsymbol{k}^{\propto}$ inherits the symplectic structure, and thus is defined the Poisson bracket over it. For two functions $H_{j}$ and $H_{k}$ over $\Theta_{A}$, it is found that

$$
\begin{aligned}
\left\{H_{j}, H_{k}\right\}(A) & =\omega_{A}\left(a d_{B_{j}}^{*} A, a d_{B_{k}}^{*} A\right) \\
= & \left(A,\left\lfloor B_{j}, B_{k}\right\rfloor\right)
\end{aligned}
$$

This equation can also be expressed as

$$
\left\{H_{j}, H_{k}\right\}(A)=\left(\left\langle B_{j}, A\right\rangle, B_{k}\right)^{g \sim g^{*}}=\left(\left[A, B_{j}\right], B_{k}\right)
$$

which means that the gradient $H_{k}(A)$ in the direction of $X_{H_{j}}$ is given by $\left\{H_{j}, H_{k}\right\}(A)$. 
c. The function $H_{j}(A) ; A \in \boldsymbol{k}^{\propto} \subset \boldsymbol{h}^{\perp} A d_{g^{-1}}^{*}$ - invariant over $\boldsymbol{g} *, \boldsymbol{g} \in \boldsymbol{G}$. This characteristic is expressed via the condition

$$
\begin{gathered}
0=\left.\frac{d}{d t} H_{j}\left(e^{-t \bar{A}} A e^{t \bar{A}}\right)\right|_{t=0}=\left(\boldsymbol{G}_{\boldsymbol{H}_{j}},[\bar{A}, A]\right), \quad \forall \bar{A} \in \boldsymbol{k}^{\propto} \\
=\left(\left\lfloor\boldsymbol{G}_{\boldsymbol{H}_{j}}, A\right], \bar{A}\right),
\end{gathered}
$$

i.e. $\left(\left[\boldsymbol{G}_{\boldsymbol{H}_{j}}, A\right], \bar{A}\right)=0 \quad$ via the nondegeneracy of $(\cdot, \cdot)$.

d. The integrability condition via Konstant-Symes theorem actually states that, if $H_{j}(A)$ and $H_{k}(A)$ are independent and $A d *$-invariant over $\boldsymbol{g} *$, then

i. $\left\{H_{j}, H_{k}\right\}=0$ over the coadjoint orbit $\boldsymbol{k}^{\propto} ; \forall j, k \in Z_{+}$,

ii. $\left\lfloor X_{H_{j}}, X_{H_{k}}\right\rfloor=0$, where the vector fields $X_{H_{j}}$ and $X_{H_{k}}$ are commutative.

The result below shows the relevancy of the algebra $g$ of the formal pseudo differential operators in GelfandDickey programme and within this group theoretical formulation.

Theorem (refer Adler [1])

Let orbit $\Theta_{p_{1}}$ via $p_{1} \in \boldsymbol{k}^{*}$ be the coadjoint action $\boldsymbol{G}$ over $\boldsymbol{k}^{*}$, that is $\Theta_{p_{1}}=\left\{\prod_{0, \infty}\left(A d_{g^{-1}}^{*} p_{1}\right) \mid g \in \boldsymbol{G}\right\}$

If $p_{1} \in a_{0, n}$, then $p_{2} \in \Theta_{p_{1}}$, that is $p_{2}=\sum_{k=0}^{n} \varphi_{k} \xi^{k}$, where the 'plane'

$$
a_{l, j}=\left\{\sum_{l \leq k \leq j} \varphi_{k} \xi^{k} \mid \varphi_{k} \in C\right\} \text { and projection } \prod_{l, j} g\left(\xi^{-1}\right) \text { onto } a_{l, j} \text { via }
$$

$\prod_{l, j}\left(\sum \varphi_{k} \xi^{k}\right)=\sum_{l \leq k \leq j} \varphi_{k} \xi^{k}$. The symplectic form, $\omega$ over $\Theta_{p_{1}}$ at $p_{2}$ is given by

$$
\begin{aligned}
\omega_{p_{2}}\left(\prod_{0, \infty}\left(a d_{q_{1}}^{*} p_{2}\right), \prod_{0, \infty}\left(a d_{q_{2}}^{*} p_{2}\right)\right) & \doteq\left(p_{2},\left[q_{1}, q_{2}\right]\right) \\
& =\left(\prod_{0, \infty}\left(a d_{q_{1}}^{*} p_{2}\right), q_{2}\right),
\end{aligned}
$$

where $q_{1}, q_{2} \in \boldsymbol{k}$, thus $H=H\left(p_{2}\right) \doteq P(\varphi) \in S\left(\boldsymbol{k}^{*}\right), \quad P(\varphi)$ a polynomial in $\varphi_{k}$ and derivatives $\varphi_{k, x^{v}}$ with respect to $x ; k=0,1, \ldots, \mathrm{n}$ and $\mathrm{v}=1,2, \ldots$, then the induced Hamiltonian vector field via $\omega$ is given

and

$$
\begin{array}{r}
X_{H}=\prod_{0, n-2}\left[G_{H}, p_{2}\right] \\
G_{H_{k^{*}}}=\sum_{j=0}^{n}(\xi+D)^{-j-1} \frac{\delta H}{\delta \varphi_{j}}
\end{array}
$$


$\frac{\delta H}{\delta \varphi_{j}}$ is the variational formal derivative with respect to $\varphi_{j}$. In addition, the formal Poisson bracket, basically from $\omega,\{\cdot, \cdot\}$, is given by

$$
\left.\left\{H_{r}, H_{s}\right\}\left(p_{2}\right) \doteq\left(p_{2}, \mid G_{H_{r}}, G_{H_{s}}\right\rfloor\right) ; \quad r, s \in Z_{+}, H_{r}, H_{s} \in S\left(\boldsymbol{k}^{*}\right)
$$

Finally, the Poisson structure is the Poisson bracket when $\varphi_{n}=1, \varphi_{n-1}=0$

where $\varphi_{k} \in C\left[\varphi_{0}, \ldots, \varphi_{n}\right] \equiv C_{n} \subset C$ and correspondingly $C_{n} / D C_{n}=I\left[\varphi_{0}, \ldots, \varphi_{n}\right]$

\section{Remark 2}

a) Conclusions extracted from the above theorem related to the form of $\Theta_{p_{1}}$, tangent space $T_{p_{2}} \Theta_{p_{1}}$, and the orbit's symplectic structure, $\omega_{p_{2}}$, are clearly derived from this group theoretical formulation (particularly from Konstant-Symes theorem). Following Gelfand-Dickey programme, $p_{2} \in \boldsymbol{k}^{\propto} \subset \boldsymbol{k}^{*} \quad, \quad$ and $p_{2}=\xi^{n}+a_{0, n-2}=\xi^{n}+\sum_{0 \leq k \leq n-2} \varphi_{k} \xi^{k}$.

b) Construction of $G_{H} \in S\left(k^{*}\right)$, associated with a localized function $H$ at $p_{2}$, i.e.

$H\left(p_{2}\right) \doteq \bar{P}(\varphi) \equiv \int P\left(\varphi_{k}, \varphi_{k, x}, \ldots, \varphi_{k, x^{\nu}}, \ldots\right) d x ; k=0,1, \ldots, n ; \quad v=1,2, \ldots$, where

$P$ is a polynomial function in $\varphi_{k}$ and derivatives $\varphi_{k, x^{v}}$ with compact support in $x$. Thus by following such characterisation, we can express $d H_{p_{2}}\left(p_{3}\right) ; p_{3} \in \Theta_{p_{1}}$ as

$$
d H_{p_{2}}\left(p_{3}\right)=\int \operatorname{Tr} \cdot\left(\frac{\delta P}{\delta \varphi_{j}} \cdot \varphi_{k}\right) d x ; j, k=0, \ldots, n
$$

$\frac{\delta P}{\delta \varphi_{j}}$ is the variational formal derivative of $P$ with respect to $\varphi_{j}$.

In Frechet sense, this becomes

$$
\begin{aligned}
d H_{p_{2}}\left(p_{3}\right) & =\left.\frac{d}{d t} H\left(p_{2}+t p_{3}\right)\right|_{t=0} \equiv\left(p_{3}, G_{H_{\mid k^{*}}}\left(p_{2}\right)\right) \\
& \text { or } \quad=\frac{d}{d t}\left(p_{2}+t p_{3}, q\right) ; \quad q \in \boldsymbol{k}, \quad \text { a functional on } \boldsymbol{k}^{*} \\
& =\left(p_{3}, q\right)
\end{aligned}
$$

This relationship can be implied to be of the form

$$
q=G_{H_{\mid k^{*}}}\left(p_{2}\right)=\sum_{j=0}^{n-2}(\xi+D)^{-j-1} \frac{\delta P}{\delta \varphi_{j}}
$$




$$
=(\xi+D)^{-1} \frac{\delta P}{\delta \varphi_{0}}+(\xi+D)^{-2} \frac{\delta P}{\delta \varphi_{1}}+\ldots,
$$

whereas the Hamiltonian vector field of the orbit structure $\Theta_{p_{1}}, X_{H}$, generated by $H$ at $p_{2}$ is given by

$$
\begin{aligned}
X_{H}\left(p_{2}\right) & =q \cdot p_{2}=a d_{q}^{*} p_{2} \in T_{p_{2}} \Theta_{p_{1}} \\
g \approx g * & \stackrel{=}{=} \prod_{0, n-2}\left[G_{H}, q\right] \\
\text { or } \quad p_{2, t} & \left.=\prod_{0, n-2}\left[G_{H}, p_{2}\right]=\prod_{-(n-1)-1,-1}\left(G_{H}\right), \prod_{0, n-2}\left(p_{2}\right)\right]
\end{aligned}
$$

i.e. the normal form of the isospectral Lax equation. $\Theta_{p_{1}}$ inherits the symplectic structure, $\omega_{p_{2}} ; p_{2} \in \Theta_{p_{1}}$, and thus defined over it a Poisson bracket; i.e. for two functions $H_{r}\left(p_{2}\right), H_{s}\left(p_{2}\right) \in \Theta_{p_{1}}$, we have

$$
\begin{gathered}
\left\{H_{r}, H_{s}\right\}\left(p_{2}\right)=\omega_{p_{2}}\left(\prod_{0, n-2}\left(a d_{q_{1}}^{*} p_{2}\right), \prod_{0, n-2}\left(a d_{q_{2}}^{*} p_{2}\right)\right. \\
\doteq\left(p_{2},\left[q_{1}, q_{2}\right]\right), \text { or can be written as } \\
\int\left(p_{2} \cdot\left[q_{1}, q_{2}\right]\right)_{-1} d x \doteq\left(p_{2},\left[G_{H_{r}}\left(p_{2}\right), G_{H_{S}}\left(p_{2}\right)\right]\right)
\end{gathered}
$$

Interestingly, by using the above results and the standard binomial identities, Adler [1] was able to show that

$$
\begin{aligned}
X_{H}\left(p_{2}\right) & =\prod_{0, n-2}\left[G_{H}, p_{2}\right] \\
= & \sum_{r=0}^{n-2}\left[\begin{array}{c}
\sum\left(\begin{array}{c}
j+\mu \\
\mu
\end{array}\right)(-D)^{\mu}\left(\frac{\delta H}{\delta \varphi_{j}} \cdot \varphi_{r+j+\mu+1}\right) \\
-\sum\left(\begin{array}{c}
r+\mu \\
\mu
\end{array}\right) \varphi_{r+j+\mu+1}(D)^{\mu} \frac{\delta H}{\delta \varphi_{j}}
\end{array}\right] \cdot \xi^{r}
\end{aligned}
$$

which is equivalent to the Gelfand-Dickey formula, i.e. the form of a KdV hierarchy, when $\varphi_{n}=1, \varphi_{n-1}=0$; i.e. in the $\boldsymbol{k}^{\propto}$ background. This indirectly validates the integration of the Gelfand-Dickey programme and the applied group theoretical approach.

c) Both equations (3) and (4) are localised with values $G_{H_{\mid k^{*}}}\left(p_{2}\right)$ at $x$ and are dependent on the values at $x$ of $\varphi_{k}$ and derivatives $\varphi_{k, x^{\nu}}$, and various variational derivatives $H$. This fact implies that the right hand sides of equations (3) and (4) are admissible without any assumptions on the compactness of $H$ and $p_{2}$; i.e. $G_{H_{\mid k^{*}}}\left(p_{2}\right)$ and $X_{H}\left(p_{2}\right)$ are rigorously defined.

d) Decisively, the KdV hierarchy acts as an Hamiltonian system over the coadjoint orbit $\Theta_{p_{1}}$, the dual space $\boldsymbol{k}^{*}$ of Lie algebra $\boldsymbol{g}$ of formal pseudo differential operator. 


\section{Integrability of the KdV hierarchies}

We have $\wp$ be the Lie algebra of the formal pseudo differential operator, $\wp=\boldsymbol{h}+\boldsymbol{k}$;

$\boldsymbol{h}=a_{0, \infty}$ (symbol algebra of non-negative type formal pseudo differential), and $\boldsymbol{k}=a_{-\infty,-1}$ (symbol algebra of negative type formal pseudo differential). $(\cdot, \cdot)$ is defined via the trace functional and becomes the symmetrical inner product, then $\wp \approx \wp^{*}=\boldsymbol{h}^{\perp}+\boldsymbol{k}^{\perp}$, where $\boldsymbol{h}^{\perp}=\boldsymbol{k}^{*}=\boldsymbol{h}, \boldsymbol{k}^{\perp}=\boldsymbol{h}^{*}=\boldsymbol{k}, a d^{*}=-a d$ with the $A d^{*}$-invariant submanifold is given by $\boldsymbol{k}^{\propto}=\left\{\xi^{n}+a_{0, n-2}\right\}$, whereas $H^{m}=\operatorname{Tr} \wp^{m / n} \in a\left(\boldsymbol{k}^{\propto}\right), m=0,1, \ldots$.

From Konstant-Symes theorem we can observe the following pertinent propositions for the KdV hierarchies.

\section{Proposition 1}

By having $p_{2}$ being extended to $p_{2} \in \boldsymbol{k}^{\propto}$, and

$$
\begin{gathered}
\boldsymbol{k}^{\propto}=\left\{\xi^{n}+\sum_{k=0}^{n-2} \varphi_{k} \xi^{k}+(\xi+D)^{k} \varphi_{k} \mid \varphi_{k} \in C\right\}, \quad n<\infty, \text { then we have } \\
G_{T r \cdot p_{2}{ }^{m+n} / n}=\left(\frac{m+n}{n}\right) \prod_{-(n-1), 0}\left(p_{2}^{m / n}\right) .
\end{gathered}
$$

Proof

Suppose $H \doteq<p_{2}^{v}>; \quad v=m / n, \quad m$ an integer and $p_{2}=\left[\lambda^{v} \varphi_{\lambda}\right]_{\left(\lambda, \xi^{n}\right)} ; \quad p_{2} \in \boldsymbol{k}^{\infty}$ and so $H \doteq<\left.\left[\lambda^{v} \varphi_{\lambda}\right]\right|_{\left(\lambda, \xi^{n}\right)}>$.

Thus $\delta H \doteq<\left[\lambda^{v} \varphi_{\lambda} \circ \delta p_{2} \circ \varphi_{\lambda}\right]_{\left(\lambda, \xi^{n}\right)}>$, where $\delta \varphi_{\lambda}=\varphi_{\lambda} \circ \delta p_{2} \circ \varphi_{\lambda}$

$$
\begin{aligned}
& \doteq\left(\left[\lambda^{v} \varphi_{\lambda}^{2}\right]_{\left(\lambda, \xi^{n}\right)}, \delta p_{2}\right) \\
& =\left(\left[-\left(\frac{\partial \varphi_{\lambda}}{\partial \lambda}\right) \lambda^{v}\right]_{\left(\lambda, \xi^{n}\right)}, \delta p_{2}\right) \\
& =\left(\left[-\left(\frac{\partial \lambda^{v}}{\partial \lambda}\right) \cdot \varphi_{\lambda}\right]_{\left(\lambda, \xi^{n}\right)}, \delta p_{2}\right) \\
& =\left(\left[v \lambda^{v-1} \varphi_{\lambda}\right]_{\left(\lambda, \xi^{n}\right)}, \delta p_{2}\right) \\
& =\left(v p_{2}^{v-1}, \delta p_{2}\right)
\end{aligned}
$$

Therefore $\nabla<p_{2}^{v}>=\left(v p_{2}^{v-1}\right)_{-(n-1), 0}$ 


\section{Proposition 2}

Let $p_{2} \in \boldsymbol{k}^{\propto}$ and $H^{m}=\operatorname{Tr} \wp^{m / n} ; m=0,1, \ldots$, then the Hamiltonian equation for the $\mathrm{KdV}$ hierarchy is of the form

$$
p_{2, t}=X_{H}\left(p_{2}\right)=\left\lfloor\prod_{-(n-1),-1}\left(G_{H^{m}}\right), \prod_{0, n-2}\left(p_{2}\right)\right\rfloor,
$$

and this implies its Lax representation as

$$
p_{2, t}=\left[q, p_{2}\right]
$$

$q$ is of the form (3) and also $q=\left(\frac{m}{n}\right) \prod_{0, \infty}\left(p_{2}^{m-n / n}\right)$

Furthermore, $H^{m}$ is in involution with respect to the Poisson bracket.

Proof

Since $p_{2}^{m / n}=\left(p_{2}^{m / n}\right)_{+}+\left(p_{2}^{m / n}\right)_{-}$and $0=\left[p_{2}^{m / n}, p_{2}\right]=\left[\left(p_{2}^{m / n}\right)_{+}+\left(p_{2}^{m / n}\right)_{-}, p_{2}\right]$.

Therefore $\left[\left(p_{2}^{m / n}\right)_{-}, p_{2}\right]=\left[p_{2},\left(p_{2}^{m / n}\right)_{+}\right]$. We thus have

$$
\begin{aligned}
& {\left[p_{2},\left(p_{2}^{m / n}\right)_{+}\right]=\left[\left(p_{2}^{m / n}\right)_{-(n-1)}, p_{2}\right]+\left[\vartheta_{-n}, p_{2}\right] ; \text { where } \vartheta_{-n} \in S_{-\infty,-n},} \\
& {\left[p_{2},\left(p_{2}^{m / n}\right)_{+}\right] \in S_{0, \infty},\left[\left(p_{2}^{m / n}\right)_{-(n-1)}, p_{2}\right] \in S_{-\infty, n-2} .}
\end{aligned}
$$

Projection onto $S_{0, \infty}$ results in

$$
\begin{aligned}
& {\left[p_{2},\left(p_{2}^{m / n}\right)_{+}\right]=\left[\left(p_{2}^{m / n}\right)_{-(n-1)}, p_{2}\right]^{n-2} } \\
& \text { Therefore }\left[p_{2},\left(p_{2}^{m / n}\right)_{+}\right]=\left[\left(\frac{n}{m+n}\right) \nabla<p_{2}^{m+n / n}>, p_{2}\right]^{n-2} \\
&= {\left[G_{H}, p_{2}\right]^{n-2} } \\
&= {\left[p_{2}, q\right] ; q=\left(p_{2}^{m / n}\right)_{+} } \\
&=p_{2, t}, \text { i.e. the Lax isospectral equation. }
\end{aligned}
$$

From the Hamiltonian formalism, we have Poisson bracket

$$
\begin{aligned}
\left\{<p_{2}^{m / n}>,<p_{2}^{k / n}>\right\} & =\left.\frac{d}{d t}\right|_{<p_{2}^{k / n}>}<p_{2}^{m / n}>; m, k \text { are integers, } \\
& =\left(p_{2, t}, \nabla<p_{2}^{m / n}>\right)
\end{aligned}
$$




$$
\begin{aligned}
& =c\left(\left[p_{2}, q\right],\left(p_{2}^{N^{\prime} / n}\right)_{-(n-1)}\right) ;\left[p_{2}, q\right] \in S_{0, n-2}, c=\frac{m}{n}, N^{\prime}=m-n \\
= & c\left(\left[p_{2}, q\right],\left(p_{2}^{N^{\prime} / n}\right)_{-}\right)=c\left(\left[p_{2}, q\right],\left(p_{2}^{N^{\prime} / n}\right)\right) \\
= & c\left(q,\left[p_{2}^{N^{\prime} / n}, p_{2}\right]\right) \\
= & 0 \text {, i.e. } H^{m} \text { is in involution w.r.t. Poisson bracket. }
\end{aligned}
$$

\section{Remark 3}

a) The results above imply that $H^{m}$ forms sets of (invariant) integral orbit in involution over $\boldsymbol{k}^{\propto}$, whereas $\boldsymbol{k}^{\propto}$ contains more than one coadjoint orbits. Moreover, $H^{m}$ is $A d^{*}$-invariant over $\boldsymbol{k}^{*}$ and comes from the statement

$$
\left[G_{H^{m}}\left(p_{2}\right), p_{2}\right]=\left[\frac{m}{n}\left(p_{2}^{m-n / n}\right), p_{2}\right]=0 .
$$

It is also obvious that, $G_{H^{m} \mid k}=\frac{m}{n} \prod_{-\infty,-1}\left(p_{2}^{m-n / n}\right)$ and $G_{H^{m} \mid k^{*}}=\frac{m}{n} \prod_{0, \infty}\left(p_{2}^{m-n / n}\right)$,

and thus via Konstant-Symes theorem we have the Lax equation (6). As a conclusion, following this perspective naturally shows the complete integrability of the KdV hierarchies.

b) From the results above, we examine the Lie geometric structure of the well-known $\mathrm{KdV}$ equation:

$$
\varphi_{t}-6 \varphi \varphi_{x}+\varphi_{x x x}=0
$$

Let $\mathrm{n}=2$ and setting $\varphi_{2}=1, \varphi_{1}=0$, appropriately with respect to Gelfand-Dickey programme, which requires that the coadjoint orbit over $\boldsymbol{k}^{\propto}$ is given with conditions that the integral orbit are invariants, i.e. $\int \varphi_{2}=$ constant, $\int \varphi_{1}=$ constant. Thus if $p_{2}=\varphi+\xi^{2} \in \boldsymbol{k}^{\propto}$ (letting $\varphi=\varphi_{0}$ ); i.e. $p_{2}$ is related to the time independent Schroedinger operator with potential $\varphi$, then via equation (3) we have

$$
\begin{aligned}
& G_{H_{j}}\left(p_{2}\right)=(\xi+D)^{-1} \frac{\delta P_{j}}{\delta \varphi}=\frac{\delta P_{j}}{\delta \varphi} \cdot \xi^{-1}-D \cdot \frac{\delta P_{j}}{\delta \varphi} \cdot \xi^{-2}+\ldots, \\
& G_{H_{k}}\left(p_{2}\right)=(\xi+D)^{-1} \frac{\delta P_{k}}{\delta \varphi}=\frac{\delta P_{k}}{\delta \varphi} \cdot \xi^{-1}-D \cdot \frac{\delta P_{k}}{\delta \varphi} \cdot \xi^{-2}+\ldots,
\end{aligned}
$$

$H_{j}, H_{k} \in a\left(\boldsymbol{k}^{\propto}\right)$ with $P_{j}, P_{k}$ are respectively polynomials to the integrals $H_{j}, H_{k}$. By employing the Lie bracket operation, we have

$$
\left[G_{H_{j}}\left(p_{2}\right), G_{H_{k}}\left(p_{2}\right)\right]=-\left(\frac{\delta P_{j}}{\delta \varphi} \cdot D \frac{\delta P_{k}}{\delta \varphi}-\frac{\delta P_{k}}{\delta \varphi} \cdot D \frac{\delta P_{j}}{\delta \varphi}\right) \xi^{-3}+\ldots
$$

This simply means that the Poisson structure on $\Theta_{p_{2}} \in \boldsymbol{k}^{\propto}$ can be written as (via (5)) 


$$
\begin{aligned}
\left\{H_{j}, H_{k}\right\}\left(p_{2}\right) & \doteq \int\left(p_{2} \cdot\left[G_{H_{j}}, G_{H_{k}}\right]\left(p_{2}\right)\right)_{-1} d x \\
= & \int-\left(\frac{\delta P_{j}}{\delta \varphi} \cdot D \frac{\delta P_{k}}{\delta \varphi}-\frac{\delta P_{k}}{\delta \varphi} \cdot D \frac{\delta P_{j}}{\delta \varphi}\right) d x \\
\text { or } & =\int\left(\frac{\delta P_{j}}{\delta \varphi} \cdot J \frac{\delta P_{k}}{\delta \varphi}\right) d x,
\end{aligned}
$$

$J$ represents the Poisson bracket.

By following the Konstant-Symes theorem and the above-mentioned theorem, this bracket is based from the symplectic structure at any coadjoint orbit in $\boldsymbol{k}^{\propto}$; i.e. we can write

$$
\omega_{p_{2}}\left(a d_{G_{H_{j}}\left(p_{2}\right)}^{*} p_{2}, a d_{G_{k}\left(p_{2}\right)}^{*} p_{2}\right)=\left\{H_{j}, H_{k}\right\}\left(p_{2}\right)
$$

Furthermore we can deduce that (via equation (4))

$$
\begin{aligned}
X_{H}\left(p_{2}\right) & =-\prod_{0, \infty}\left[p_{2}, G_{H}\left(p_{2}\right)\right]=-\prod_{0, \infty}\left[\varphi+\xi^{2}, G_{H}\left(p_{2}\right)\right] \\
= & -\prod_{0, \infty}\left[\xi^{2}, \frac{\delta P}{\delta \varphi} \cdot \xi^{-1}\right],
\end{aligned}
$$

the other terms are ignored since these are of negative degree. If we assume that there exists an algebraic isomorphism between the 'formal pseudo differential operator space' and 'symbol space', then

$$
X_{H}\left(p_{2}\right)=-2 D \frac{\delta P}{\delta \varphi}
$$

If $P=P_{3}=\varphi^{3}+\frac{1}{2} \varphi_{x}^{2}$ and $D=\partial_{x}$, then the vector fields for the orbit $\Theta_{p_{2}}$ structure is given by

$$
X_{H}=\left(6 \varphi \varphi_{x}-\varphi_{x x x}\right) \partial \varphi
$$

evolution field of the KdV (7).

From the results above and together with the flow generated by $X_{H}$ (coadjoint action of $G$ on $\boldsymbol{k}^{*}$ ), definitively we can conclude that the dynamical system related to $\mathrm{KdV}$ is a Hamiltonian mechanical system over the coadjoint orbits in $\boldsymbol{k}^{\propto}$.

\section{Concluding Remarks}

We have shown briefly that the above group theoretical formulation exhibits a coherent connection between Lax representation and mechanics on the coadjoint orbit space of a Lie group action. Clearly this framework is largely based on sterling works done by Adler [1], Lebedev \& Manin [15] and Berezin \& Perelomov [4], which asserts firstly that the relevant symplectic structure is the symplectic structure of the relevant orbit and secondly the integrability of the KdV hierarchy's dynamical system and its Lax representation are closely related to the Lie algebra splitting. This manifestation naturally explains the integrability of the KdV hierarchies via this group theoretical approach. In fact, we think that the approach is also applicable to other general class of nonlinear partial differential equations (eg. Drinfeld [7], Semenov-Tian-Shansky [20], Terng \& Uhlenbeck [23]). We believe (as Palais [17]) that the secret sources of soliton symmetries and as well as the many remarkable 
properties of soliton equations are closely related to the existence of large and non-obvious groups of symplectic automorphisms. These groups would act on the phase spaces of these Hamiltonian systems (the coadjoint orbit space of a Lie group action) and leave the Hamiltonian function invariant. Furthermore, this framework simply brings forth further understanding with respect to the link between (affine) Lie algebra representations and integrable systems.

The concept of integrability, which is being extended to infinite dimensions or to partial differential equation, is just a portion of a rich structure found in the class of 'completely integrable' equations or the 'soliton' equations residing within the integrable systems (as Batlle [3]). The 'unreasonable effectiveness' of these full-blown structures can be seen in the remarkable recent emergence of a most exotic range of actively researched disciplines: two dimensional quantum field theory, intersection theory on the moduli space of Riemann surfaces, integrable hierarchies, matrix integrals, random surfaces, Gromov-Witten invariants, and many more (eg. Chen et al [5], Dijkgraaf [6], Marshankov [16]).

\section{References}

[1] M. Adler, On a trace functional for formal pseudo-differential operators and the symplectic structure for the Korteweg-de Vries type equations, Invent. Math., 50 (1979), 219-248.

[2] M. Adler and P. van Moerbeke, Completely integrable systems, Kac-Moody Lie algebras and curves, $A d v$. Math., 38 (1980), 267-317.

[3] C. Batlle, Lecture notes on $\mathrm{KdV}$ hierarchies and pseudodifferential operators, Geometria Diferencial I Aplicacions, MAT-GDA/92/1, (1992)

[4] F.A. Berezin and A.M. Perelomov, Group theoretic interpretation of the Korteweg-de Vries type equations, Commun. Math. Phys., 74 (1980), 129-140.

[5] Y. Chen, M. Kontsevich and A. Schwarz, Symmetries of WDVV equations, arXiv:hep- th/0508221 v1 (2005).

[6] R. Dijkgraaf, Intersection Theory, Integrable Hierarchies and Topological Field Theory, in New Symmetry Principles in Quantum Field Theory (Cargese, 1991), NATO Adv. Sci. Inst. Ser. B Phys., 295, Plenum, New York, 1992, 95-158.

[7] V.G. Drinfeld, Hamiltonian structures on Lie groups, Lie bialgebras and the geometric meaning of classical Yang-Baxter equations, Sov. Math. Doklady 27 (1983), 68-71.

[8] V.G.Drinfeld and V.V. Sokolov, Lie algebras and equations of Korteweg-de Vries type, Soviet Math., 30 (1985), 1975-2036.

[9] Frenkel, Affine Kac-Moody algebras, integrable systems and their deformations, Hermann Weyl Prize Lecture, XXIV Colloquium on Group Theoretical Methods, Paris, July 172002.

[10] I.M. Gelfand and L.A. Dickey, Fractional powers of operators and Hamiltonian systems, Funct. Anal. Appl., 10 (1976), 259-273.

[11] I.M. Gelfand and L.A. Dickey, The resolvent and Hamiltonian systems, Funct. Anal. Appl., 10 (1977), 259-273.

[12] A.A. Kirillov, Elements of the Theory of Representations, Springer-Verlag, New York, 1976.

[13] B.Konstant, The solution to a generalised Toda lattice and representation theory, Ad. Math., 34 (1979), 195-338.

[14] P.D. Lax, Integrals of nonlinear equations of evolution and solitary waves, Comm. Pure. Appl Math. 21 (1968), 467-490.

[15] D.R. Lebedev and Yu I. Manin, Gelfand-Dikii Hamiltonian operator and the coadjoint representation of the Volterra group, J. Soviet Math., 13 (1980), 268-273.

[16] Marshakov, Strings, Integrable Systems, Geometry and Statistical Models, arXiv:hep-th/0401199 v2, (2004).

[17] R.S. Palais, The Symmetries of Solitons, Bull. Amer. Math. Soc., 34 (1997), 339-403. 
[18] Sato, A remark on the connection between affine Lie algebras and soliton equations, Lett. Math.Phys., 16 (1988), 125-132.

[19] R. Schmid, (2004) Infinite dimensional Lie groups with applications to mathematical physics, Geo. Symmetry in Physics, 1 (2004) 1-67.

[20] M.A. Semenov-Tian-Shansky, (1987). Classical r-matrices, Lax equations, Poisson Lie group actions, Lecture Notes in Physics, Springer-Verlag, vol. 280 (1987) 174-214.

[21] J.M. Souriau, Structure des systemes dynamiques, Dunod, Paris, 1970.

[22] W. Symes, Hamiltonian group actions and integrable systems, Physica D, 1 (1980), 339-374.

[23] C.L. Terng and K. Uhlenbeck, Geometry of Solitons, Notices of AMS, Vol. 47 No.1, (2000), 17-25.

[24] Weinstein, The local structure of Poisson manifolds, J. Diff. Geo., 18 (1983), 523-557. 